\title{
Acculturation and Filial Piety as Mediators of the Relationship Between Caregiver Burden and Gender-Role Expectations in Hispanic-American Rehabilitation Services Students
}

Roy K. Chen

University of Texas Rio Grande Valley, roy.chen@utrgv.edu

Bryan S. Austin

University of Idaho, Boise, baustin@uidaho.edu

Chien-Chun Lin

Western Oregon University, linc@mail.wou.edu

Follow this and additional works at: https://nsuworks.nova.edu/ijahsp

Part of the Community Psychology Commons, Counselor Education Commons, Health Psychology Commons, and the Rehabilitation and Therapy Commons

\section{Recommended Citation}

Chen RK, Austin BS, Lin C. Acculturation and Filial Piety as Mediators of the Relationship Between Caregiver Burden and Gender-Role Expectations in Hispanic-American Rehabilitation Services Students. The Internet Journal of Allied Health Sciences and Practice. 2017 Jan 01;15(4), Article 5.

This Manuscript is brought to you for free and open access by the College of Health Care Sciences at NSUWorks. It has been accepted for inclusion in Internet Journal of Allied Health Sciences and Practice by an authorized editor of NSUWorks. For more information, please contact nsuworks@nova.edu. 


\title{
Acculturation and Filial Piety as Mediators of the Relationship Between Caregiver Burden and Gender-Role Expectations in Hispanic-American Rehabilitation Services Students
}

\begin{abstract}
Purpose: Hispanic-Americans are the largest ethnic minority group in the United States. The need to care for older Hispanics has become an important issue as they now enjoy longer life expectancies due to better access to healthcare and less labor participation in hazardous occupations. The present study examined whether the association between caregiver burden and gender-role expectations is mediated by acculturation and filial piety. Method: The sample consisted of 93 Mexican-American rehabilitation services students enrolled at a large public university in Texas. The four instruments used in the study were the Zarit Burden Interview, the Bem Sex-Role Inventory, the Acculturation Rating Scale for MexicanAmericans-Revised, and the Expectations of Filial Piety Scale. Participants visited an online survey site to respond to the questionnaire. Results: About half of the most common person to whom care was being provided was a parent $49.5 \%(n=46)$ with $35.5 \%(n=33)$ grandparents and $15.1 \%(n=14)$ siblings. Acculturation scores were positively correlated with both gender-role expectations $(r=.23, p=.027)$ and filial piety $(r=.30, p=.003)$ scores. In addition, caregiver burden scores were negatively correlated with gender-role expectation scores $(r=-.21, p=.046)$ and filial piety scores were positively correlated with gender-role expectation scores $(r=.29, p=.005)$. The correlation between caregiver burden and filial piety was not statistically significant $(r=-.10, P>.05$ n.s.). Moreover, neither acculturation nor filial piety mediated the relationship between gender-role expectations and caregiver burden. Conclusion: MexicanAmericans view aging positively, have a sense of caring for elders, and have strong ties to their culture. As a result of strong Hispanic family values, the obligation to care for family members (i.e., familism) tends to occur with little hesitance. Future replication studies are needed to better understand the effects of acculturation and filial piety on caregiver burden among Mexican-Americans residing in other regions of the country, including outside the cities along the United States-Mexico border. The rationale for further research is that living in predominantly-White states such as Minnesota and Wyoming, where MexicanAmericans make up a small fraction of the population, might have effects on their acculturation.
\end{abstract}

\section{Author Bio(s)}

Roy K. Chen, PhD, CRC, is a professor of rehabilitation counseling in the School of Rehabilitation Services and Counseling at the University of Texas Rio Grande Valley.

Bryan S. Austin, PhD, LPC, CRC, is an assistant professor of rehabilitation counseling at the University of Idaho, Boise.

Chien-Chun Lin, PhD, CRC, is an assistant professor of rehabilitation counseling at Western Oregon University.

Acknowledgements

N/A

This manuscript is available in Internet Journal of Allied Health Sciences and Practice: https://nsuworks.nova.edu/ijahsp/vol15/iss4/5 


\title{
IJAHSP \\ The Internet Joumnal of Allied Health Sciences and Practice \\ Dedicated to allied health professional practice and education
}

Vol. 15 No. 4 ISSN 1540-580X

\section{Acculturation and Filial Piety as Mediators of the Relationship between Caregiver Burden and Gender-Role Expectations in Hispanic-American Rehabilitation Services Students}

Roy K. Chen, $\mathrm{PhD}^{1}$

Bryan S. Austin, PhD²

Chien-Chun Lin, $\mathrm{PhD}^{3}$

1. University of Texas Rio Grande Valley

2. University of Idaho, Boise

3. Western Oregon University

United States

\begin{abstract}
Purpose: Hispanic-Americans are the largest ethnic minority group in the United States. The need to care for older Hispanics has become an important issue as they now enjoy longer life expectancies as a result of better access to healthcare and less labor participation in hazardous occupations. The present study examined whether the association between caregiver burden and gender-role expectations is mediated by acculturation and filial piety. Method: The sample consisted of 93 MexicanAmerican rehabilitation services students enrolled at a large public university in Texas. The four instruments used in the study were the Zarit Burden Interview, the Bem Sex-Role Inventory, the Acculturation Rating Scale for Mexican-Americans-Revised, and the Expectations of Filial Piety Scale. Participants visited an online survey site to respond to the questionnaire. Results: About half of the most common person to whom care was being provided was a parent $49.5 \%(n=46)$ with $35.5 \%(n=33)$ grandparents and $15.1 \%(n=14)$ siblings. Acculturation scores were positively correlated with both gender-role expectations $(r$ $=.23, p=.027)$ and filial piety $(r=.30, p=.003)$ scores. In addition, caregiver burden scores were negatively correlated with gender-role expectation scores $(r=-.21, p=.046)$ and filial piety scores were positively correlated with gender-role expectation scores $(r=.29, p=.005)$. The correlation between caregiver burden and filial piety was not statistically significant $(r=-.10, P$ $>.05$ n.s.). Moreover, neither acculturation nor filial piety mediated the relationship between gender-role expectations and caregiver burden. Conclusion: Mexican-Americans view aging positively, have a sense of caring for elders, and have strong ties to their culture. As a result of strong Hispanic family values, the obligation to care for family members (i.e., familism) tends to occur with little hesitance. Future replication studies are needed to better understand the effects of acculturation and filial piety on caregiver burden among Mexican-Americans residing in other regions of the country, including outside the cities along the United States-Mexico border. The rationale for further research is that living in predominantly-White states such as Minnesota and Wyoming, where Mexican-Americans make up a small fraction of the population, might have effects on their acculturation.
\end{abstract}

\section{INTRODUCTION}

The racial hues of the United States (U.S.) have steadily changed over the past decades. Of the 53 million Hispanic-Americans, the largest ethnic minority group in the country, more than $65 \%$ of them self-identify as Mexican-Americans. ${ }^{1}$ The U.S. Census Bureau projects the growth of the Hispanic population to reach 128.8 million by the year $2060 .{ }^{2}$ Geographically, HispanicAmericans concentrate mainly in California, Florida, Texas, New York, and Illinois, where employment opportunities are more abundant. For example, Hispanic-Americans account for $38.2 \%$ of the entire population in the state of Texas in $2014 .{ }^{2}$

In general, the Hispanic population in the U.S. has not only grown markedly in numbers but also prolonged their longevity. As a result of advancement in medicine, better access to healthcare, and less labor participation in hazardous occupations, HispanicAmericans as a group now enjoy longer life expectancies. ${ }^{3}$ The need to care for older Hispanics, therefore, has become an 
important issue. In a recent study of Hispanic family caregivers, the findings revealed that one-third of families surveyed had at least one family caregiver, and almost three-quarters of them were females with an average age of 43 years old. ${ }^{4}$ In addition, about $5 \%$ of Hispanic caregivers were students, which was significantly higher than across all ethnicity groups. ${ }^{4}$ Notably, Hispanic-American family caregivers reported having stronger senses of familism and also found more fulfillment in providing care than their White counterparts. ${ }^{4}$ Researchers believe that the cultural background of a caregiver could influence how he or she defines the caregiving experience; that is, the perceived burden on an individual to care for a family member who is ill or has a disability. ${ }^{5}$

\section{Acculturation}

Acculturation occurs when people of different cultural heritages, religious values, and social mores come in contact, whether by choice or not, with each other within a society as they strive to attain a harmonious co-existence by adopting new blended behaviors and attitudes. ${ }^{6}$ Although the process of being acculturated influences all groups, such said changes nevertheless tend to have more impact on minority groups than the majority culture. ${ }^{7}$ Some acculturation indicators commonly observed in the U.S. include nativity status, generational status, length of residency, and English acquisition. ${ }^{8}$

Researchers have found acculturative stress to be a key factor in causing higher levels of psychological distress and anxiety among Hispanic adolescents and young adults. ${ }^{9}$ Second-generation immigrants are more prone to feel caught between traditional values inherited from their parents and their own values of the host country. ${ }^{9}$ The degree of acculturation also determines the pattern of caregiving rendered in Hispanic families. Specifically, individuals who have a strong sense of familism are more dutiful to provide long-term care themselves to a family member in need of care than to rely on outside help. ${ }^{10}$ However, another plausible explanation posited by researchers is that such caregiving arrangements may be made out of necessity and often represent the only viable option for many less acculturated Hispanic-Americans. Such Hispanic-Americans may identify with a lower socioeconomic bracket due to their legal residency status and limited English proficiency, and consequently, are ineligible for Medicaid and other governmental assistance programs. ${ }^{11}$

\section{Gender Roles}

In the paternalistic Mexican family structure, the primary roles expected of women are that of mothers and caregivers. ${ }^{12}$ Typically, Hispanic stay-at-home caregivers are older daughters and/or daughters-in-law, who oftentimes are less educated, unemployed, and in poorer physical and psychological health than White family caregivers. ${ }^{11}$ It is not unusual for Mexican-American families to adhere to the rigid, stereotypical gender-role expectations and demand women to quit their jobs in order to fulfill their caregiver roles. ${ }^{5}$ Yet, such inflexible decisions are sometimes plagued with many unintended adverse consequences at the expense of female caregivers' well-being. For example, prior research has documented Mexican-American caregivers having suffered from loss of income, escalating inter-generational conflicts, lower caregiver satisfaction over time, and increasing sleep disturbance and fatigue. ${ }^{5}$

Although first-generation Mexican immigrant women might still abide closely to the beliefs of traditional gender-roles, the secondand third-generation American-born daughters and granddaughters may hold somewhat different views as they experience greater amounts of exposure to mainstream values and are encouraged to pursue opportunities outside their home settings. ${ }^{13}$ Despite the continued loosening of the long-held gender-role expectations, Pinto and Coltrane concluded that most family caregivers of Mexican origin, regardless of generational status or traditional gender roles, choose to stay home to provide care because of practical concerns such as time constraints and scarce resources. ${ }^{12}$

\section{Filial Piety}

Filial piety is a family-laden value deeply ingrained in the ancient philosophy of Confucianism widely practiced in East Asia. ${ }^{14}$ The personal obligation to respect and care for elders, particularly aging parents, is the epitome of filial piety. ${ }^{3}$ MexicanAmericans understand and relate to this concept as shown by the strong bond prevalent within their close-knit family units. Filial piety is a ubiquitous cultural norm of Mexican-American families, which not only provides positive meanings to care for family members, but also mitigates caregiver stressors. ${ }^{15}$

In a study of 286 Mexican-American college students in south Texas, Rudolph et al found no gender differences in filial responsibility expectations, although female caregivers rendered the bulk of the care at home. ${ }^{16}$ However, another study pointed out the extent of acculturation in Mexican-Americans impinges on their perceptions of filial responsibility and attitudes within the value of familism. ${ }^{3}$ By contrast, the authors also indicated that filial piety is viewed less as a burden in Mexican-American families than the White participants. Taking care of family members is considered more of a normal part of their lives in the Mexican-

(c) The Internet Journal of Allied Health Sciences and Practice, 2017 
American community. Furthermore, Funk et al discussed the relationships between filial obligation and well-being of caregivers. ${ }^{15}$ The authors noted that caregivers who have high-level senses of filial piety but unable to provide caregiving, would report feelings of guilt. Paradoxically, caregivers who have low senses of filial obligations may endure higher incidences of stress and various health conditions. This phenomenon, as the authors suggested, is worth further examination on how the level of filial responsibility could affect caregiver well-being.

\section{Familism}

Familism is a highly revered traditional value espoused in a collectivistic society such as the Mexican-American community. For instance, early on, children are raised to be loyal, respectful, and supportive of the family. It is quite common for family members to provide both material and emotional backing to each other, particularly to their elders. ${ }^{7}$ John, Resendiz, and De Vargas wrote that Mexican-Americans view family as their primary source of identity and draw support from family especially in the time of crisis. ${ }^{17}$ Furthermore, Mexican-Americans believe that the needs and welfare of the family are an essential priority and should supplant all other needs of individual family members. These cultural norms about familial responsibility are routinely passed down through generations and hence infuse sturdily into their worldview.

The profound impact the changing economy has had on the cohesiveness of Mexican-American family units and their traditional values cannot be understated. Such shifts in attitudes and mindsets may be inevitable as more Mexican-Americans of the new generations work outside of their hometowns and have to adopt new behaviors and values which often make providing care to other family members more difficult and less feasible in the Mexican-American community. ${ }^{17}$ According to Herrera et al, there is a negative correlation between acculturation and familism. In other words, higher levels of acculturation were significantly related to lower levels of familism. ${ }^{11}$ This claim is further corroborated by the study of Crist et al in which more acculturated MexicanAmericans (i.e., high on individualism) tended to exhibit less familism (i.e., low on collectivism). ${ }^{7}$ Crist et al speculated that high caregiver burden could be attributed to low familism because new generations of Mexican-Americans might view themselves as grudgingly bounded to their assumed caregiver roles. ${ }^{7}$ One silver lining amidst the much transformed Mexican-American community is that they are still more committed to living close to parents and caring for them when sick than White-Americans. ${ }^{3}$

\section{Caregiver Burden}

Caregiving is an arduous task which can be both physically taxing and psychologically draining to the provider. In contrast to popular opinion, caregiving should not frivolously be seen as free labor or service when calculated as part of the economics matrix of opportunity cost. Simply put, the provider must forgo the ability of earning an income in order to stay home to care for the sick or disabled family member, with little or no financial compensation in return. Hoenig and Hamilton succinctly described the dichotomous nature of burden in two aspects: objective and subjective. ${ }^{18}$ The objective impacts are definitive effects on the household, health, financial loss, and daily chores; whereas, the subjective impacts are the perceptions of caregivers about the burden they feel. Oftentimes, in addition to the aforementioned issues, the burden of care also includes stressful feelings such as shame, embarrassment, resentment, feelings of guilt, and self-blame. ${ }^{19}$

Prior research has linked caregiver burden to an array of variables, such as the kind of impairments, behavioral problems, functional limitations, and type and amount of care and supervision demanded by individuals with disabilities. ${ }^{19,20}$ Particularly, time committed to performing care tasks is a reliable predictor of the caregiver's stress, anger, burnout, and poor psychological and physical health. ${ }^{21}$ Nonetheless, Mexican-Americans in general are quite accepting of the disability status of their family members. ${ }^{19}$ For example, the presence of strong family ties helps stabilize and assuage the conditions of Mexican-Americans with schizophrenia. ${ }^{22}$

When examining the difference in the magnitude of caregiver burden between Hispanic- and White-Americans, Jenkins and Schumacher concluded that gender, ethnicity, diagnosis of disability, and living situation all played a vital role. ${ }^{23}$ Magana, Ramirez-Garcia, Hernandez, and Cortez estimated between $12 \%$ and $18 \%$ of Mexican-American family caregivers were at high risk of suffering from depression. ${ }^{24}$ And among this at-risk population, those providers who were younger in age, with lower educational attainment, and had care recipients with severe mental illness, were susceptible to develop depressive symptoms themselves. Herrera et al noted Mexican-Americans not only prefer to give and receive care in their own homes than other choices, but also had lower utilization of external long-term care than other ethnicity groups. ${ }^{10}$ These culturally influenced decisions therefore make Hispanic-American caregivers face unique challenges. ${ }^{15}$ Given the complexity of caregiver burden issues, the purpose of this study was to examine how acculturation and filial piety mediated the relationship between caregiver burden and expectations of gender-role in Mexican-American families. Specifically, caregiver burden refers to the caring for family members with a disability or chronic illness.

(C) The Internet Journal of Allied Health Sciences and Practice, 2017 


\section{METHOD}

\section{Participants}

The sample comprised of 93 Mexican-American students. The majority of the participants were female $(n=74,79.6 \%)$. The most common levels of education were graduate students $(n=41,44.1 \%)$, followed by juniors $(n=23,24.7 \%)$, and seniors $(n$ $=23,24.7 \%)$, with only $2.2 \%(n=2)$ freshmen and $4.3 \%(n=4)$ sophomores. About half of the most common person to whom care was being provided was a parent $49.5 \%(n=46)$, with $35.5 \%(n=33)$ grandparents and $15.1 \%(n=14)$ siblings. The age of the participants ranged from 18 to 57 years old, with a mean of 26.85 years old $(s d \pm 8.51)$. The duration of time as a caregiver ranged from one month to 24 years, with a mean of 5.04 years $(s d \pm 5.10)$.

\section{Measures}

Caregiver Burden. The construct of caregiver burden was measured by the 22 -item Zarit Burden Interview $(\mathrm{ZBI}) .{ }^{25}$ Each item is ranked on a 5-point Likert type scale from $0=$ never, $1=$ rarely, $2=$ sometimes, $3=$ quite frequently, to $4=$ nearly always. The total possible score ranges from 0 to 88. A high score correlates with higher level of burden. The Cronbach's a for the present study was .931 .

Gender-Role Expectations. The construct of gender-role expectations was assessed by the short version of the Bem Sex-Role Inventory (BSRI). ${ }^{26}$ The BSRI contains 30 items measuring 10 masculine and 10 feminine characteristics. Each item is ranked on a 7-point Likert type scale from $1=$ not applicable to $7=$ totally applicable. The 10 filler items are excluded from the computation of personality traits. The total scores were computed as T scores (with a mean of 50 and standard deviation of 10) based on femininity minus masculinity, meaning that higher scores (above 50) were indicative of a more feminine orientation and lower scores (below 50) were indicative of a more masculine orientation. The Cronbach's a for masculinity and femininity of the present study were .808 and .902 , respectively.

Acculturation. The construct of acculturation was assessed by the 30-item Acculturation Rating Scale for Mexican-AmericansRevised (ARSMA-II). ${ }^{27}$ Each item is ranked on a 5-point Likert type scale from $1=$ not at all, $2=$ very little/not very often, $3=$ moderately, $4=$ much/very often, to $5=$ extremely often/almost always. The total possible score ranges from 30 to 150. A high score correlates with a higher level of acculturation. The Cronbach's a for the present study was .862 .

Filial Piety. The construct of filial piety was measured by the 16 -item Expectations of Filial Piety Scale (EFPS). ${ }^{28}$ Each item is scored on a 4-point Likert type scale from $1=$ no need at all, $2=$ somewhat in need, $3=$ pretty much in need, to $4=$ very much in need. The total possible score ranges from 16 to 64 . A high score correlates with a higher level of filial piety. The Cronbach's a for the present study was .828 .

\section{Procedure}

This study was conducted at a large public university located in Texas. Upon receiving approval from the Institutional Review Board at the University of Texas Rio Grande Valley, the first author contacted prospective participants. The research parameters included students who were enrolled in undergraduate and graduate degree seeking programs while at the same time were caregivers at home for their parents, grandparents, or siblings with a disability or chronic illness. Surveys excluded students who care for their own children with disabilities. Participants were recruited by obtaining permission from course instructors who announced this study to their students across multiple courses. Students who indicated interest were given a flyer, which provided a URL link to an online survey site.

Figure 1 depicts the proposed model tested in the current study to investigate the mediating effects of acculturation and filial piety on the relationship between gender-role expectations and caregiver burden. Z scores were used to check the significance of skewness and kurtosis of the variables. The Levene's test was selected to check the assumption of homogeneity of variance. The distributions were further examined by applying the Kolmogorov-Smirnov test. A series of regression analyses were used to test the mediating effects. ${ }^{29,30}$ The Sobol test was also used to examine the statistical significance of the indirect effects. 31,32 


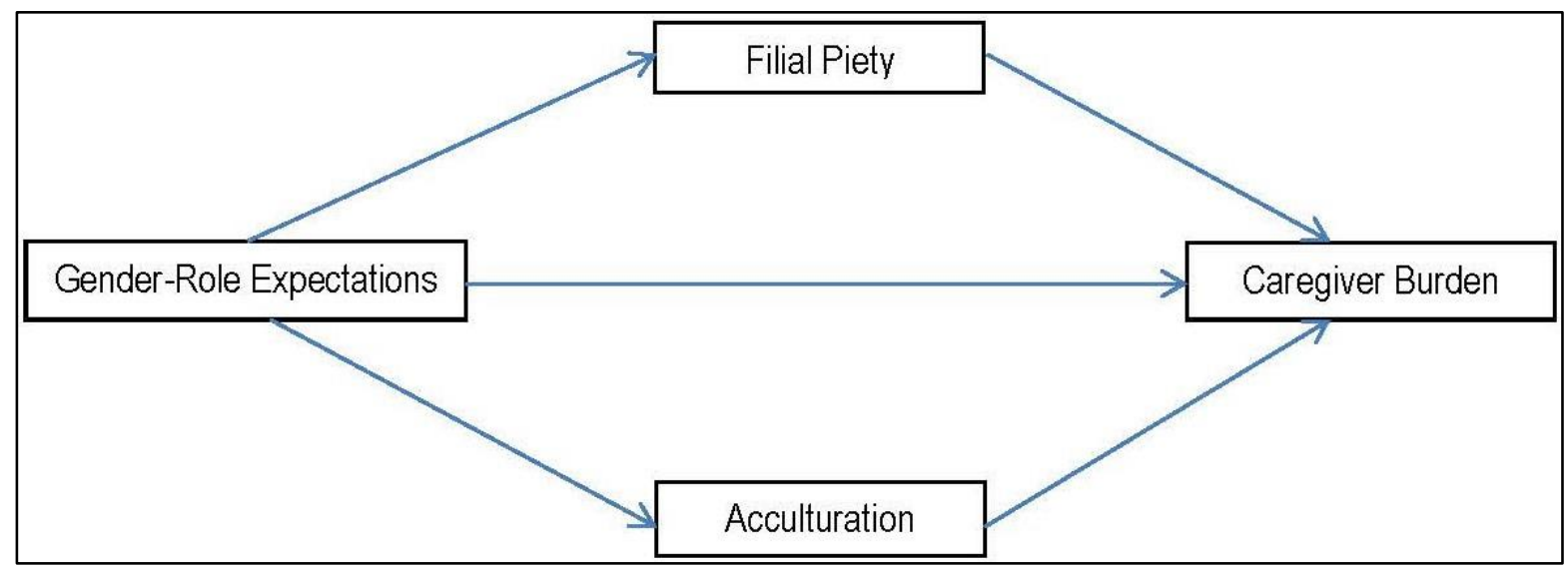

Figure 1. Mediating effects of acculturation and filial piety on the relationship between gender-role expectations and caregiver burden.

\section{RESULTS}

Descriptive statistics for the composite variables in this study are as follows: caregiver burden $(m=55.41, s d \pm 15.17$, range $=$ 26 to 94 ), gender-role expectations $(m=55.39, s d \pm 10.72$, range $=26$ to 79$)$, filial piety $(m=48.25, s d \pm 7.15$, range $=26$ to 63 ), and acculturation $(m=109.18, s d \pm 13.94$, range $=63$ to 138). Acculturation scores were positively correlated with both gender-role expectations $(r=.23, p=.027)$ and filial piety $(r=.30, p=.003)$ scores. In addition, caregiver burden scores were negatively correlated with gender-role expectation scores $(r=-.21, p=.046)$ and filial piety scores were positively correlated with gender-role expectation scores $(r=.29, p=.005)$. However, the correlation between caregiver burden and filial piety was not statistically significant $(r=-.10, p>.05$, non-significant.).

\section{Mediation Tests}

In all of the mediation analyses, gender, age, level of education, years of taking care of family member with a disability, and recipient of care were used as control variables. Gender was entered as a dummy variable coded as $0=$ male, $1=$ female. Age and years of taking care of disabled family member were entered in their original continuous form. Educational attainment was recoded as $0=$ undergraduate and $1=$ graduate student. For the recipient of care, there were three possible values: grandparents, parents, and siblings. Therefore, two dummy variables were created for grandparents (coded as 1 if the caredfor person was a grandparent and 0 if not) and siblings (coded as 1 if the cared-for person was a sibling and 0 if not), with parents as the reference category.

The mediation analyses were performed using the procedures outlined by Baron and Kenny. ${ }^{29}$ The first step in this mediationtesting procedure is to establish that the independent variable (gender-role expectations) is predictive of the mediator variables (acculturation and filial piety). Tables 1 and 2 show the results from these regression analyses. When gender-role expectation scores were used as a predictor of acculturation scores, the regression model was statistically significant, $F(7,85)=4.04, p=$ .001 . The $R^{2}$ of .25 indicated that $25 \%$ of the variance in acculturation was explained in this model. Three of the control variables were statistically significant including age $(\beta=-.24, p=.034)$, years as a caregiver $(\beta=.32, p=.002)$, and recipient of care $(\beta=$ $-.25, p=.026$ for grandparents and $\beta=-.35, p=.001$ for siblings). These results indicated that acculturation scores were highest for younger participants, participants who had been caregivers for a longer period of time, and for caregivers of parents as opposed to grandparents or siblings. 
Table 1. Results from Regression Analysis with Gender-Role Expectations as a Predictor of Acculturation ( $N=93$ )

\begin{tabular}{|l|c|c|c|c|c|}
\hline Predictor & $\boldsymbol{B}$ & $\boldsymbol{S} E_{\boldsymbol{B}}$ & $\boldsymbol{\beta}$ & $\boldsymbol{t}$ & $\boldsymbol{p}$ \\
\hline & & & & & \\
\hline (Constant) & 111.09 & 9.40 & & 11.82 & $<.001$ \\
\hline Gender & -2.58 & 3.34 & -.08 & -.77 & .441 \\
\hline Age & -.39 & .18 & -.24 & -2.16 & .034 \\
\hline Education & -.99 & 2.86 & -.04 & -.34 & .731 \\
\hline Years as a caregiver & .87 & .27 & .32 & 3.23 & .002 \\
\hline Recipient of care & & & & & \\
\hline$\quad$ Grandparent & -7.33 & 3.24 & -.25 & -2.26 & .026 \\
\hline Sibling & -13.66 & 4.03 & -.35 & -3.39 & .001 \\
\hline Gender-role expectations & .21 & .13 & .16 & 1.61 & .112 \\
\hline \multicolumn{2}{|l|}{} & & & & \\
\hline
\end{tabular}

Notes. $R^{2}=.25, F(7,85)=4.04, p=.001$.

The primary effect of interest in this model was the effect of gender-role expectations on acculturation. This effect was not statistically significant $(\beta=.16, p>.05)$. This indicated that when the control variables were included in the model, gender-role expectations did not have an effect on acculturation. However, the $p$-value for this effect approached statistical significance, and therefore, the remaining tests for mediation were conducted with the caveat that the independent variable did not have a statistically significant effect on the mediator variable.

The results from the regression analysis for the second mediator variable (filial piety) are shown in Table 2. This regression model was not statistically significant, $F(7,85)=1.53, p>.05$. The $R^{2}$ of .11 indicated that $11 \%$ of the variance in filial piety was explained in this model. None of the control variables were statistically significant in this model (i.e., none of the demographic and background variables were related to filial piety). The independent variable, gender-role expectations, was statistically significant, $\beta=.30, p=.007$. This indicated that participants with more feminine gender-role expectations (i.e., those with higher scores on the BSRI scale) tended also to have higher scores on the filial piety scale. Thus, the first condition for mediation was met for the filial piety mediator variable.

Table 2. Results from Regression Analysis with Gender-Role Expectations as a Predictor of Filial Piety ( $\mathrm{N}=93$ )

\begin{tabular}{|l|c|c|c|c|c|}
\hline Predictor & $\boldsymbol{B}$ & $\mathbf{S E}_{\boldsymbol{B}}$ & $\boldsymbol{\beta}$ & $\boldsymbol{t}$ & $\boldsymbol{p}$ \\
\hline & & & & & \\
\hline (Constant) & 37.03 & 5.25 & & 7.06 & $<.001$ \\
\hline Gender & -2.09 & 1.86 & -.12 & -1.12 & .266 \\
\hline Age & .06 & .10 & .08 & .63 & .530 \\
\hline Education & -.32 & 1.60 & -.02 & -.20 & .843 \\
\hline Years as a caregiver & .09 & .15 & .07 & .61 & .543 \\
\hline Recipient of care & & & & & \\
\hline Grandparent & -.43 & 1.81 & -.03 & -.24 & .812 \\
\hline Sibling & .19 & 2.25 & .01 & .08 & .933 \\
\hline Gender-role expectations & .20 & .07 & .30 & 2.76 & .007 \\
\hline
\end{tabular}

Notes. $R 2=.11, F(7,85)=1.53, p=.169$

The next step in the mediation-testing procedure of Baron and Kenny is to show that the independent variable (gender-role expectations) is predictive of the dependent variable (caregiver burden) when the mediator variables are not included in the analysis. ${ }^{29}$ Table 3 shows the results from this model. This regression model was statistically significant, $F(7,85)=2.23, p=$ .039. The $R^{2}$ of .16 indicated that $16 \%$ of the variance in caregiver burden was explained in this model. The only statistically significant control variable was the recipient of care with both grandparent caregivers $(\beta=-.30, p=.014)$ and sibling caregivers $(\beta=-.23, p=.036$ ) having lower caregiver burden scores than caregivers of parents. In addition, gender-role expectation scores were statistically significant in this regression model, $\beta=-.29, p=.007$. This indicated that those with more feminine gender-role expectations (i.e., those with higher scores on gender-role expectations scale) tended to have lower scores on caregiver burden scale. Thereby, the second condition for mediation, that the independent variable be predictive of the dependent variable, was met. 
Table 3. Results from Regression Analysis with Gender-Role Expectations as a Predictor of Caregiver Burden ( $\mathrm{N}=93)$

\begin{tabular}{|l|c|c|c|c|c|}
\hline Predictor & $\boldsymbol{B}$ & $\boldsymbol{S} \boldsymbol{E}_{\boldsymbol{B}}$ & $\boldsymbol{\beta}$ & $\boldsymbol{t}$ & $\boldsymbol{p}$ \\
\hline & & & & & \\
\hline (Constant) & 81.69 & 10.85 & & 7.53 & $<.001$ \\
\hline Gender & 4.46 & 3.86 & .12 & 1.16 & .251 \\
\hline Age & -.07 & .21 & -.04 & -.31 & .758 \\
\hline Education & -1.89 & 3.30 & -.06 & -.57 & .569 \\
\hline Years as a caregiver & .04 & .31 & .01 & .11 & .910 \\
\hline Recipient of care & & & & & \\
\hline$\quad$ Grandparent & -9.40 & 3.75 & -.30 & -2.51 & .014 \\
\hline$\quad$ Sibling & -9.90 & 4.65 & -.23 & -2.13 & .036 \\
\hline Gender-role expectations & -.41 & .15 & -.29 & -2.75 & .007 \\
\hline & & & & & \\
\hline
\end{tabular}

Notes. $R^{2}=.16, F(7,85)=2.23, p=.039$.

The third and final step in the mediation-testing procedure of Baron and Kenny is to examine the change in prediction of the dependent variable (caregiver burden) by the independent variable (gender-role expectations) when the mediator variables (acculturation and filial piety) are included in the model. ${ }^{29}$ Table 4 shows the results from this model. The overall model was not statistically significant, $F(9,83)=1.82, p>.05$. The $R^{2}$ coefficient indicated that $16 \%$ of the variance in caregiver burden was explained in this model. Among the control variables, only the recipient of care was statistically significant, with caregivers of both grandparents $(\beta=-.33, p=.010)$ and siblings $(\beta=-.27, p=.024)$, having lower caregiver burden scores than caregivers of parents.

Table 4. Results from Regression Analysis with Gender-Role Expectations, Acculturation, and Filial Piety as a Predictor of Caregiver Burden ( $\mathrm{N}=93)$

\begin{tabular}{|l|c|c|c|c|c|}
\hline Predictor & $\boldsymbol{B}$ & $\boldsymbol{S} E_{\boldsymbol{B}}$ & $\boldsymbol{\beta}$ & $\boldsymbol{t}$ & $\boldsymbol{p}$ \\
\hline & & & & & \\
\hline (Constant) & 95.56 & 18.41 & & 5.19 & $<.001$ \\
\hline Gender & 4.11 & 3.92 & .11 & 1.05 & .297 \\
\hline Age & -.11 & .22 & -.06 & -.50 & .617 \\
\hline Education & -2.01 & 3.33 & -.07 & -.61 & .547 \\
\hline Years as a caregiver & .14 & .33 & .05 & .42 & .675 \\
\hline Recipient of care & & & & & \\
\hline$\quad$ Grandparent & -10.27 & 3.88 & -.33 & -2.64 & .010 \\
\hline$\quad$ Sibling & -11.51 & 5.01 & -.27 & -2.30 & .024 \\
\hline Acculturation & -.12 & .13 & -.11 & -.90 & .370 \\
\hline Filial piety & -.02 & .23 & -.01 & -.09 & .928 \\
\hline Gender-role expectations & -.38 & .16 & -.27 & -2.43 & .017 \\
\hline & & & & & \\
\hline
\end{tabular}

Notes. $R^{2}=.16, F(9,83)=1.82, p=.077$.

Neither of the mediator variables were statistically significant in this model as predictors of caregiver burden. In addition, genderrole expectations were still statistically significant in this regression model, $\beta=-.27, p=.017$. Compared to the model without the two mediators (shown in Table 3), this represents a reduction from only -.29 to -.27 in the effect of gender-role expectation scores. This indicated that mediation of the effect of gender-role expectations on caregiver burden by either acculturation or filial piety did not occur in this study.

In addition to testing the specific conditions for mediation outlined by Baron and Kenny, ${ }^{29}$ the Sobol test was used to examine the statistical significance of the indirect effects. ${ }^{31,32}$ Two indirect effects were examined. The first indirect effect was GenderRole Expectations $\rightarrow$ Acculturation $\rightarrow$ Caregiver Burden. The calculation of the Sobol test indicated that this indirect effect was not statistically significant, $z=-.80, p>.05$. This indicated that gender-role expectation scores did not have an indirect effect on caregiver burden scores through acculturation scores and is consistent with the conclusion that acculturation did not mediate the effect of gender-role expectations on caregiver burden. 
The second indirect effect examined was Gender-Role Expectations $\rightarrow$ Filial Piety $\rightarrow$ Caregiver Burden. The Sobol test for this indirect effect was also not statistically significant, $z=-.09, p>.05$. This showed that there was no indirect effect of gender-role expectations on caregiver burden through filial piety, which again is consistent with the conclusion that filial piety was not a mediator of the relationship between gender-role expectations and caregiver burden.

\section{DISCUSSION}

The purpose of the present study was to examine how acculturation and filial piety mediated the relationship between caregiver burden and expectations of gender-role in Mexican-American families. There were two key findings from this study. First, the results showed that gender-role expectations were predictive of caregiver burden, with participants having a more feminine gender-role expectation also tending to have lower caregiver burden. The second conclusion was that neither acculturation nor filial piety mediated the relationship between gender-role expectations and caregiver burden. Past findings support the present research. ${ }^{33,34}$ For instance, Phillips et al found that female Mexican-Americans viewed aging positively, had a sense of caring for elders, and had stronger ties to the Mexican-American culture. ${ }^{34}$ Caregiving duties may be perceived positively by Hispanic caregivers as a result of their strong family-oriented values, and therefore, may further explain why Mexican-Americans feel well-adjusted and not overly burdened while caring for their older family members. ${ }^{33}$

Conversely, the finding that filial piety and acculturation were not statistically significant, and therefore, did not have a mediating effect on the relationship between gender-role expectations and caregiver burden, was unexpected, particularly because expectations of females to care for their family/loved ones appears to be intimately tied to the Mexican-American culture. ${ }^{11,35}$ This finding also contrasts the researchers' hypothesis and prior research that links cultural factors to caregiving outcomes.3,36 For example, Rudolph et al concluded that filial responsibility and individualistic values were significant and important acculturation effects on Mexican-American caregiver role expectations. ${ }^{3}$ Similarly, Kao and Kyungeh found acculturation linked to family loyalty for Mexican-American caregivers who held more traditional Mexican culture orientations. ${ }^{36}$ Despite this conflicting research, the reasons why acculturation and filial piety did not show a mediating effect in the current study can be understood in several ways. Maybe strong Hispanic family values, such as obligation to care for their own family members (i.e., familism), occurs without hesitance. To illustrate, perhaps the expectation for Mexican-American females to take a leading role of caring for family members has been passed on through generations. ${ }^{35}$ Instead of understood as a duty or obligation, caregiving may be associated with positive feelings [e.g., pride, cohesion with family unit] and "deeply rooted in the cultural subconsciousness, arising 'naturally' without conscious thought." $35($ p.3) This interpretation is consistent with research findings about Mexican-Americans and their caregiver experiences. ${ }^{34}$

It is also likely that change in filial norms among different geographic sub-regions and -populations within the Mexican-American culture needs further exploration to help better understand within-group acculturation value differences. ${ }^{3}$ Mexican-Americans may be less acculturated in certain geographic regions across the U.S. For example, while only $67 \%$ of residents in South Texas are Hispanic, the number in the Lower Rio Grande Valley exceeds $90 \% .{ }^{37}$ Thus, acculturation and pressure to adopt more mainstream White-American values may be more or less relevant for some Mexican-Americans over others. ${ }^{34}$ In addition, the filial piety construct used for this study may not be culturally sensitive. ${ }^{38,39}$ As a result, filial piety, a concept rooted in Confucianism, may not register with many Mexican-Americans. ${ }^{40}$

\section{Limitations}

In light of findings of the current study, the results should be considered within the context of their limitations. Sampling bias may have been introduced as study participants were not randomly selected. The small sample size may also curtail the interpretation of findings to only Mexican-American caregivers in the Rio Grande Valley. It may be that a different sample of Mexican-American participants might have responded differently. All participants were students at an institution of higher education and may hold divergent caregiver attitudes than caregivers who are not going to school, as past research has shown caregivers who have less education tend to experience higher burden. ${ }^{41}$ Furthermore, participants were mostly younger, and therefore, may yield different results than an older, aged sample of Mexican-Americans.

\section{Implications for Rehabilitation Professionals}

The caregiver burden research further reinforces the need for cultural sensitivity and multicultural competencies among rehabilitation professionals and the importance of focusing attention to caregivers who provide care and support to family members with disabilities. ${ }^{42,43}$ Effectively addressing the needs of caregivers has potential to enhance the quality of patient care rehabilitative services, and ultimately, lead to positive outcomes for both clients and their caregivers. ${ }^{44}$ The present study adds to the accumulating evidence in this area and provides empirical support for rehabilitation counseling and related allied health

(C) The Internet Journal of Allied Health Sciences and Practice, 2017 
professions (e.g., occupational therapy, physical therapy) to address perceived culturally relevant gender-role expectations as they relate to caregiver burden for Mexican-American caregivers. ${ }^{38,44}$ Therefore, in response to findings for this study, the following recommendations for rehabilitation counselors and related rehabilitation professionals who provide counseling and/or rehabilitation services to Mexican-American family caregivers are highlighted below.

Include family caregiver in assessment and rehabilitation planning. Rehabilitation counselors and related allied health professionals should consider including Mexican-American family caregivers in addition to the patient with a disability by assessing the family as part of an individual client assessment. ${ }^{33}$ Past research combined with findings from this study suggest that Mexican-American family members with more feminine gender-role expectations are generally less burdened when providing care to their family members. ${ }^{3}$ Even though this may be the case, many Mexican-American caregivers still experience burden, and thus, psychological distress (e.g., depressive symptoms, burnout) that can affect their continuing ability to provide high quality care and support.45

Assess acculturation and gender roles. Although neither acculturation nor filial piety was found to have a mediating effect on the relationship between gender-role expectations and caregiver burden for Mexican-American caregivers in the current study, the research indicates that within-group differences likely exist. ${ }^{3}$ Consequently, if a Mexican-American caregiver is more (or less) assimilated to White-Americanized values and/or identifies with more (or less) feminine roles, this knowledge may lead to new insights for interpreting a caregiver's burden more precisely, and thereby, how to best intervene from a Mexican-American cultural perspective. To ensure Mexican-American family and caregiver cultural values are uniquely appraised for each family unit, rehabilitation professionals should give thought to selecting cultural-specific constructs (i.e., familismo, machismo) ${ }^{46}$ and instruments normed and validated with Mexican-American populations (e.g., Acculturation Rating Scale for Mexican-AmericansRevised).27,38 In addition, interventions intended to help Mexican-American patients and their caregivers further explore the meaning of their unique family caregiving experiences from each of their own cultural frames of reference may have added value. 40

\section{Recommendations for Future Research}

Taking into account this study's findings, there are a number of recommendations for future caregiver research in rehabilitation counseling and related allied health professions. Purposeful sampling of Mexican-Americans from various geographic regions may give a fuller picture of cultural similarities and differences across the U.S. and should be pursued. Given the variance of measures used to measure filial values and need to better understand constructs in their complexity, other measures, not the focus of this study, should also be studied in their own right and as mediating between gender-role and caregiver burden for Mexican-American families, such as religion, family loyalty, and mutuality, among other variables. ${ }^{11,36}$ It may also be that other related instruments are better suited for measuring Mexican-American cultural norms [i.e., filial piety]. ${ }^{40}$ Alternatively, care recipient perspectives as well as the dyadic caregiver-care recipient relationship may be another important angle to the study of caregiver processes and outcomes. Hence, future caregiving research is needed to add to this knowledge base because outcomes (e.g., depressive symptoms) for family caregivers and care recipients (patient family members with a disability) may be intricately linked. ${ }^{44}$

\section{References}

1. Pew Research Center. Hispanic Trends, 2015.Retrieved from http://www.pewhispanic.org/2008/02/11/us-populationprojections-2005-2050/

2. United States Census Bureau, 2014. Retrieved from http://www.census.gov/data.html

3. Rudolph B, Cornelius-White C, Quintana F. Filial responsibility among Mexican American college students: A pilot investigation and comparison. J Hispanic High Educ. 2005;4(1):64-78. doi:10.1177/1538192704271667

4. National Alliance for Caregiving and Evercare. Evercare study of Hispanic family caregiving in the U.S.: Findings from a national study, 2008. Retrieved from

http://www.caregiving.org/data/Hispanic_Caregiver_Study_web_ENG_FINAL_11_04_08.pdf

5. Cagle CS, Wolff E. Blending voices of Mexican American cancer caregivers and healthcare providers to improve care. Oncol Nurs Forum. 2009;36(5):555-62. [PMID: 19726395]

6. Schwartz SJ, Unger JB, Zamboanga BL, Szapocznik J. Rethinking the concept of acculturation: Implications for theory and research. Am Psychol. 2010;65(4):237-51. doi:10.1037/a0019330 [PMID: 20455618]

7. Crist JD, McEwen MM, Herrera AP, Kim SS, Pasvogel A, Hepworth JT. Caregiving burden, acculturation, familism, and Mexican American elders' use of home care services. Res Theory Nurs Pract. 2009;23(3):165-80. doi:10.1891/15416577.23.3.165 [PMID: 19769211]

(c) The Internet Journal of Allied Health Sciences and Practice, 2017 
8. Cox RB Jr, Roblyer MZ, Merten MJ, Shreffler KM, Schwerdtfeger KL. Do parent-child acculturation gaps affect early adolescent Latino alcohol use? A study of the probability and extent of use. Subst Abuse Treat Prev Policy. 2013;8(4). doi:10.1186/1747-597X-8-4 [PMID: 23347822]

9. Crockett LJ, Iturbide MI, Torres Stone RA, McGinley M, Raffaelli M, Carlo G. Acculturative stress, social support, and coping: Relations to psychological adjustment among Mexican American college students. Cultur Divers Ethnic Minor Psychol. 2007;13(4):347-55. doi:10.1037/1099-9809.13.4.347 [PMID: 17967103]

10. Herrera AP, Lee JW, Nanyonjo RD, Laufman LE, Torres-Vigil I. Religious coping and caregiver well-being in MexicanAmerican families. Aging Ment Health. 2009;13(1);84-91. doi:10.1080/13607860802154507 [PMID: 19197693]

11. Herrera AP, Lee J, Palos G, Torres-Vigil I. Cultural influences in the patterns of long-term care use among Mexican American family caregivers. J Appl Gerontol. 2008;27(2):141-65. doi:10.1177/0733464807310682

12. Pinto KM, Coltrane S. Divisions of labor in Mexican origin and Anglo families: Structure and culture. Sex Roles. 2009;60(78):482-95. doi:10.1007/s11199-008-9549-5

13. Blau FD, Kahn LM. The gender pay gap: Have women gone as far as they can? Acad Manag Perspect. 2007;21(1):7-23.

14. Chen RK, Jo SJ, Donnell C. Enhancing the rehabilitation counseling process: Understanding the obstacles to Asian Americans' utilization of services. J Appl Rehabil Couns. 2004;35(1):29-35.

15. Funk LM, Chappell NL, Liu G. Associations between filial responsibility and caregiver well-being: Are there differences by cultural group? Res Aging. 2013;35(1):78-95. doi:10.1177/0164027511422450

16. Rudolph B, Chavez M, Quintana F, Salinas G. Filial responsibility expectations among Mexican American undergraduates: Gender and biculturalism. J Hispanic High Educ. 2011;10(3):168-82. doi:10.1177/1538192710397732

17. John R, Resendiz R, De Vargas LW. Beyond familism? Familism as explicit motive for eldercare among Mexican American caregivers. J Cross Cult Gerontol. 1997;12(2):145-62. [PMID: 14617933]

18. Hoenig J, Hamilton MW. Elderly psychiatric patients and the burden on the household. Psychiatr Neurol. 1966;152(2):28193. [PMID: 5966211]

19. Awad AG, Voruganti LN. The burden of schizophrenia on caregivers. PharmacoEconomics. 2008;26(2):149-62. [PMID: 18198934]

20. Dowdell EB. Grandmother caregivers and caregiver burden. Am J Matern Child Nurs. 2004;29(5):299-304. [PMID: 15329631]

21. East PL, Weisner TS. Mexican American adolescents' family caregiving: Selection effects and longitudinal associations with adjustment. Fam Relat. 2009;58(5):562-77. [PMID: 24000269]

22. Breitborde NJK, López SR, Chang C, Kopelowicz A, Zarate R. Emotional over-involvement can be deleterious for caregivers' health: Mexican Americans caring for a relative with schizophrenia. Soc Psychiatry Psychiatr Epidemiol. 2009;44(9):716-23. doi:10.1007/s00127-008-0492-0 [PMID: 19190836]

23. Jenkins JH, Schumacher JG. Family burden of schizophrenia and depressive illness: Specifying the effects of ethnicity, gender and social ecology. Br J Psychiatry. 1999;174(1):31-8. doi:10.1192/bjp.174.1.31

24. Magaña SM, Ramírez-García JI, Hernández MG, Cortez R. Psychological distress among Latino family caregivers of adults with schizophrenia: The roles of burden and stigma. Psychiatr Serv. 2007;58(3):378-84. [PMID: 17325112]

25. Zarit SH, Reever KE, Back-Peterson J. Relatives of the impaired elderly: Correlates of feelings of burden. Gerontologist. 1980;20(6):649-55. doi:10.1093/geront/20.6.649 [PMID: 7203086]

26. Bem SL. A Manual for the Bem Sex Role Inventory. Palo Alto, CA: Consulting Psychologist Press; 1981.

27. Cuellar I, Arnold B, Maldonado R. Acculturation Rating Scale for Mexican Americans-II: A revision of the original ARSMA Scale. Hisp J Behav Sci. 1995;17(3):275-304. doi:10.1177/07399863950173001

28. Kao SH, McHugh ML, Travis SS. Psychometric tests of Expectations of Filial Piety Scale in a Mexican-American population. J Clin Nurs. 2007;6(8):1460-7. doi:10.1111/j.1365-2702.2006.01639.x

29. Baron RM, Kenny DA. The moderator-mediator variable distinction in social psychological research: Conceptual, strategic, and statistical considerations. J Pers Soc Psychol. 1986;51(6):1173-82. doi:10.1037/0022-3514.51.6.1173 [PMID: 3806354]

30. Miles J, Shevlin M. Applying Regression and Correlation: A Guide for Students and Researchers. Thousand Oaks, CA: SAGE Publications; 2010.

31. Sobel ME. Asymptotic confidence intervals for indirect effects in structural equation models. Sociol Methodol. 1982;13(1):290-312. doi:10.2307/270723

32. Sobel, ME. Some new results on indirect effects and their standard errors in covariance structure models. In: Tuma NB, ed. Sociological Methodology. San Francisco, CA: Josey-Bass;1986:159-86.

33. Friedmann M-L, Buckwalter KC. Family caregiver role and burden related to gender and family relationships. J Fam Nurs. 2014;20(3):313-36. [PMID: 24777069] 
34. Phillips LR, Torres de Ardon E, Komnenich P, Killeen M, Rusinak R. The Mexican American caregiving experience. Hisp J Behav Sci. 2000;22(3):296-313.

35. Pharr JR, Francis CD, Terry C, Clark MC. Culture, caregiving, and health: Exploring the influence of culture on family caregiver experiences. ISRN Public Health. 2014;1-8. doi:10.1155/2014/689826

36. Kao H-F S, Kyungeh A. Effect of acculturation and mutuality on family loyalty among Mexican American caregivers of elders. J Nurs Scholarsh. 2012;44(2):111-9. [PMID: 22497839]

37. United States Census Bureau. Texas State Department Populations Projection data, 2008. Retrieved from http://greatertexasfoundation.org/wp-content/uploads/2011/03/South-Texas-Short-Research-Loop-FINAL.pdf

38. Dilworth-Anderson P, Williams IC, Gibson BE. Issues of race, ethnicity, and culture in caregiving research: A 20-year review (1980-2000). Gerontologist. 2002;42(2):237-72. [PMID: 11914467]

39. Kao H-FS, Travis SS. Effects of acculturation and social exchange on the expectations of filial piety among Hispanic/Latino parents of adult children. Nurs Health Sci. 2005;7(4):226-34. [PMID: 16271128]

40. Jones PS, Winslow BW, Lee JW, Burns M, Zhang XE. Development of a caregiver empowerment model to promote positive outcomes. J Fam Nurs. 2011;17(1):11-28. [PMID: 21343620]

41. Lahaie C, Earle A, Heymann J. An uneven burden: Social disparities in adult caregiving responsibilities, working conditions, and caregiver outcomes. Res Aging. 2013;35(3):243-74.

42. Austin BS, Leahy MJ. Construction and validation of the clinical judgment skill inventory: Clinical judgment skill competencies that measure counselor debiasing techniques. Rehabil Res Policy Educ. 2015;29(1):27-46.

43. Barros-Bailey M. Ethics and the family in rehabilitation counseling. J Rehabil. 2015;81(2):32-8.

44. Martire LM, Lustig AP, Schulz R, Miller GE, Helgeson VS. Is it beneficial to involve a family member? A meta-analysis of psychosocial interventions for chronic illness. Health Psychol. 2004;23(6):599-611. [PMID: 15546228]

45. Rote S, Angel JL, Markides K. Health of elderly Mexican American adults and family caregiver distress. Res Aging. 2015;37(3):306-31. [PMID: 25651573]

46. Falicov CJ. Mexican families. In: McGoldrick M, Giordano J, Pearce JK. eds. Ethnicity and Family Therapy, 2e. New York, NY: The Guilford Press;1996:169-82. 